\title{
The role of dielectric constant in sodium chloride solution chemistry: Magnitude of super saturation
}

\author{
Mutasim I. Khalii and Saud I. Al-Resayes \\ Department of Chemistry, Faculty of Science, King Saud University, P. O. Box 2455, Riyadh 11451, Saudi Arabia. \\ Accepted 4 January, 2012

\begin{abstract}
The effect of ethanol addition to aqueous semi saturated $\mathrm{NaCl}$ solutions on the super saturation was studied. The amount of precipitated salt was found to increase as the percent of added ethanol increased. The rates of formation of precipitate were calculated and found to increase with decrease of the weighted average of the solvents mixture dielectric constant, $\varepsilon$, but levels out at $\varepsilon \approx 50$. The solubility of salt was then connected with the dielectric constant and a zero solubility was observed at $\varepsilon$
\end{abstract} \\ $=35$. A magnitude of super saturation was defined for the measurement of solubility.
}

Key words: Dielectric constant, precipitate, activation energy, magnitude of super saturation.

\section{INTRODUCTION}

Crystallization of sodium chloride was investigated in several works (Birchall and Davey, 1981; Mohameed et al., 1997; Kubota et al., 2000; Al-Jubbouri and Ulrich, 2001, 2002; Doki et al., 2002; Linnikov, 2004; 2006a, b; 2007). It was reported that the overall growth, dissolution rates and the surface integration step are linear with respect to supersaturation of solution (Mohameed et al., 1997; Al-Jubbouri and Ulrich, 2001, 2002). Linnikov (2007) investigated the spontaneous crystallization of sodium chloride from aqueous ethanol solutions, reported that the use of ethanol for inducing super saturation in solutions allowed varying strongly the crystallization process conditions. Doki et al. (2002) crystallized sodium chloride from aqueous solution by adding pure ethanol (99 vol.\% ethanol) or an ethanol-water (75- vol.\% ethanol mixture as diluents).

The kinetics of spontaneous crystallization of potassium and sodium chlorides have been extensively studied by Linnikov $(2004,2007)$, who determined the growth rates and activations energies and found that the earlier was proportional to supersaturation. He described the kinetics of the observed crystals aggregation by the famous Smoluchowski equation for coagulation of colloid particles. Smoluchowski (1917) and Linnikov (2006a, b)

${ }^{*} \overline{C o r r e s p o n d i n g ~ a u t h o r . ~ E-m a i l: ~ m k h a l i i @ k s u . e d u . s a . ~}$ indicated that the coalescence of crystals at the spontaneous crystallization of sodium chloride from supersaturated aqueous ethanol solutions is due to formation of contacts of coalescence in the form of nucleus-bridges according to the theory of Polak (1966). However, a decrease in aggregation constant was observed as super saturation is lowered. A model of spontaneous crystallization process was then proposed (Linnikov, 2006b). The crystal size distribution and their weighed fraction in a crystal deposit of the salt were determined approximately.

Such finding could be argued in terms of the solvent dielectric constant which is a measure of orientational correlation between molecules. In fact, it is one characteristic of great importance in causing ion formation by a solute. It could be defined in terms of the medium's ability to regulate the force between two ions. Hence, the degree of solute ionization, precipitation and coagulation could be varied by varying the dielectric constant of the medium.

This has a great impact on experimental applications to chemical problems in the sense that the procedures of materials building (from bottom to top) and separation (or ionization) (from top to bottom) could be manipulated, attained and controlled by varying the medium dielectric constant.

In this paper, we present the relation between the average dielectric constant of the aqueous-ethanol mixture 
Table 1. Comparison between calculated and experimental dielectric constants.

\begin{tabular}{|c|c|c|c|}
\hline \multicolumn{2}{|c|}{ Solvent (\%) } & \multirow{2}{*}{$\begin{array}{c}(\varepsilon) \\
\text { Calculated }\end{array}$} & \multirow{2}{*}{$\varepsilon$ Experimenta } \\
\hline $\mathrm{H}_{2} \mathrm{O}$ & $\mathrm{C}_{2} \mathrm{H}_{5} \mathrm{OH}$ & & \\
\hline Pure & - & 80.0 & 79.04 \\
\hline- & Pure & 24.6 & 23.6 \\
\hline 83.3 & 16.6 & 70.9 & 68.8 \\
\hline 50 & 50 & 52.4 & 51.2 \\
\hline 20 & 80 & 35.7 & 35.4 \\
\hline
\end{tabular}

and the solubility of $\mathrm{NaCl}$ and velocity of crystal formulation. A measurement of induced super saturation, that is, the magnitude of super saturation is suggested and calculated to account for the induced supersaturation.

\section{EXPERIMENTAL PROCEDURE}

A $23.6 \%$ sodium chloride aqueous stock solution was prepared and stored in a $500 \mathrm{~cm}^{3}$ volumetric flask. The density of this stock solution was estimated by averaging the mass of different volumes, that is, 1,5 and $10 \mathrm{~cm}^{3}$ and by calculation.

A set of six experiments was then carried out as follows: $5.0 \mathrm{~cm}^{3}$ stock solution was transferred into each of six $50.0 \mathrm{~cm}^{3}$ beakers to which 1.0, $2.05 .0,10.0,15.0$ and $20.0 \mathrm{~cm}^{3}$ of $96 \%$ ethanol were then added successively stirred and allowed to reach equilibrium at ambient temperature $\left(27^{\circ} \mathrm{C}\right)$, then filtered. Caution was exercised to transfer the precipitate. The precipitates, dried at $120^{\circ} \mathrm{C}$, were then weighed and the amount of sodium chloride left in solution was calculated by difference.

The average dielectric constant of the aqueous-ethanol solvent mixture was estimated by the relation:

$$
\sum \frac{V_{i} \varepsilon_{i}}{V_{T}}=\varepsilon_{T}
$$

where $\mathrm{V}_{i}$ and $\varepsilon_{i}$ are the volume and dielectric constant of solvent $i$, respectively; and $\mathrm{V}_{\mathrm{T}}$ is the total volume of solution. The validity of Equation (1) was tested by comparing the calculated dielectric constant with the experimental one using WYNE KERR precision component analyser-6440 B. The relative dielectric constant $\left(\varepsilon_{\mathrm{r}}\right)$ was calculated by Equation (2):

$\varepsilon_{r}=\frac{C_{x}}{C_{0}}$

where $C_{x}$ is the capacitance of dielectric and $C_{0}$ is the capacitance of test capacitor when vacuum is between its plates $\left(C=8.85^{\star} 10 \mathrm{~F}\right)$.

\section{RESULTS AND DISCUSSION}

The capacitance $\left(\mathrm{C}_{\mathrm{x}}\right)$ of dielectrics was measured applying a wide range of frequencies $(20 \mathrm{~Hz}-30 \mathrm{MHz})$.

The reported values in Table 1 are for $0.3 \mathrm{kHz}$ at $\approx$ $32^{\circ} \mathrm{C}$. The trend of dielectric lowering as percent of ethanol increases is in agreement with recent report for benzene and nitrobenzene mixture Ramana and Malakondaiah (2010). However, detailed study will be published.

In dealing with the solubility of the alkali metal salts, for example, $\mathrm{NaCl}$, it is the ion-dipole interactions that are the main source of the solvation energy of the alkali metal ions. With negative ion, dipole forces are the major solvent interaction taking place. Such a fact is rationalized by applying coulomb's law in the form:

$$
\text { Force }=\frac{q_{1} q_{2}}{r^{2} \varepsilon}
$$

where $r$ is the distance between the two charges $q_{1}$ and $\mathrm{q}_{2}$ and $\varepsilon$ is the dielectric constant. It then goes without saying that the force of interaction between the charges, is inversely proportional to the dielectric constant, $\varepsilon$. One may assume that the solubility of salts is connected with dielectric constant of the medium. This is the same as saying addition of ethanol $\left(\varepsilon=24.6^{\prime}\right)$ to an aqueous solution $(\varepsilon=80)$ create supersaturation allowing to vary strongly the crystallization process conditions (Doki et al., 2002).

The results obtained show that the solubility of sodium chloride is directly proportional to $\varepsilon_{T}$ or inversely proportional to ethanol percent (volume of ethanol added /total volume of solution) ${ }^{\star} 100$ ] in mixture in accordance with the earlier argument (Table 2 and Figures $1 a$ and $b$ ). The initial and final concentrations of solutions are calculated from the number of dissolved $\mathrm{NaCl}$ moles.

The average growth rate of crystal $\mathrm{V}$ could be calculated using Linnikov (2006a,b) proposed equation:

$\mathrm{V}=833.22(\mathrm{Mi}-\mathrm{Mf}) \cdot \exp \left(\frac{-E_{a} \times 10^{3}}{R T}\right)$

where $E_{a}$ is the activation energy of growth process $\left(\mathrm{kJ} \cdot \mathrm{mol}^{-1}\right), \quad \mathrm{M}_{\mathrm{i}}$ and $\mathrm{M}_{\mathrm{f}}$ are the initial and final concentrations shown in Table 2. The activation energy of growth process of $\mathrm{NaCl}$ used in this calculation is 49.1 $\mathrm{kJ} \mathrm{mol}^{-1}$ (Linnikov, 2006a). Table 3 and Figures $2 \mathrm{a}$ and $\mathrm{b}$ show the values calculated. Here we assume that Equation (4) is valid for all percentages of alcohol. It is evident then that an inverse correlation between the velocity growth rate and the weighted dielectric constant is only valid at high values of the dielectric constant, that is, above 52.0, or, expressed in other words, directly proportional to ethanol percent in the solution mixture. This is in near agreement with the linear dependence of the growth rate with respect to supersaturation of solution reported (Linnikov, 2006b). However it is evident that such a velocity remains almost constant at above $50 \%$ ethanol, that is, below a dielectric constant value of $\approx 52.0$.

The magnitude of supersaturation, which is a measure 
Table 2. Calculated data of dielectric constant by Equation 1 and solubility of $\mathrm{NaCl}$ results.

\begin{tabular}{|c|c|c|c|c|c|c|c|}
\hline No. & $\begin{array}{c}\text { Volume of } 23.6 \% \\
\mathrm{NaCl} \text { solution } \\
(\mathrm{ml})^{*}\end{array}$ & $\begin{array}{c}\text { Volume of } 96 \% \\
\text { ethanol added } \\
(\mathrm{ml})^{*}\end{array}$ & $\begin{array}{l}\text { Vol. } \% \text { of } \\
\text { ethanol in } \\
\text { solution }\end{array}$ & $\begin{array}{l}\text { Dielectric constant } \\
\text { of solvent mixture* }\end{array}$ & $\begin{array}{l}\text { Initial concentration } \\
\text { of solution } \\
\left(\mathrm{M}, \mathrm{mol} \mathrm{I}^{-1}\right)\end{array}$ & $\begin{array}{c}\text { Final concentration } \\
\text { of solution } \\
\left(\mathrm{M}_{\mathrm{f}}, \mathrm{mol} \mathrm{I}^{-1}\right)\end{array}$ & $\begin{array}{c}\mathrm{NaCl} \\
\text { solubility } \\
(\mathrm{g} / 100 \mathrm{~g})\end{array}$ \\
\hline 1 & 5.0 & 0.00 & 0.00 & 80.0 & 4.03 & 4.03 & 30.94 \\
\hline 2 & 5.0 & 1 & 16.66 & 70.9 & 3.358 & 2.958 & 22.70 \\
\hline 3 & 5.0 & 2 & 28.57 & 64.3 & 2.878 . & 2.289 & 17.57 \\
\hline 4 & 5.0 & 5 & 50.00 & 52.4 & 2.015 & 1.157 & 8.82 \\
\hline 7 & 5.0 & 20 & 80.00 & 35.72 & 0.805 & 0.046 & 0.353 \\
\hline
\end{tabular}

${ }^{*}$ Density of $23.6 \% \mathrm{NaCl}=1.169 \mathrm{~g} \cdot \mathrm{ml}^{-1}$; density of $96 \%$ ethanol $=0.81 \mathrm{~g} \cdot \mathrm{ml}^{-1}$ dielectric constant of: $\mathrm{H}_{2} \mathrm{O}=80.0$; ethanol = 24.6.

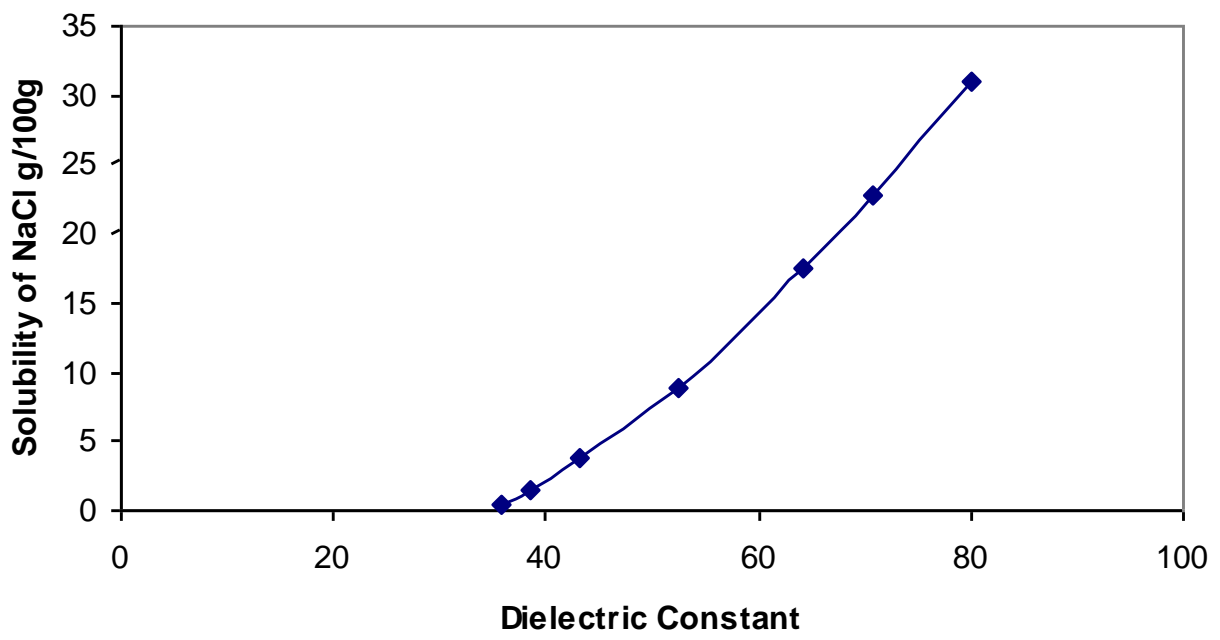

Figure 1a. Solubility of $\mathrm{NaCl}$ versus dielectric constant of the aqueous-ethanol solvent mixture.

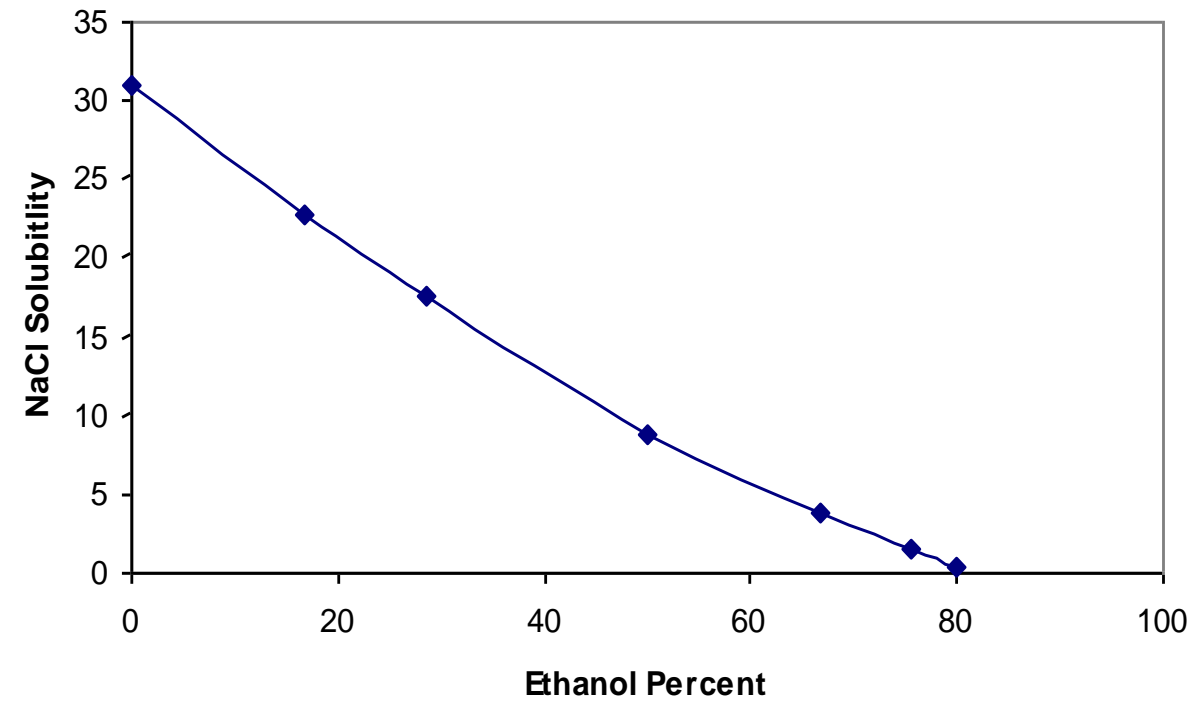

Figure 1b. Ethanol percent in aqueous-methanol solvent mixture versus $\mathrm{NaCl}$ solubility. 
Table 3. Calculated velocity of $\mathrm{NaCl}$ growth by Equation (4) versus ethanol percent and dielectric constant of aqueous-ethanol solvent mixture.

\begin{tabular}{|c|c|c|c|}
\hline No. & Ethanol (\%) & Dielectric constant & Velocity $\times 10^{11}\left(\mathrm{~m} \cdot \mathrm{s}^{-1}\right)$ \\
\hline 1 & 0 & 80.0 & 0.00 \\
\hline 2 & 16.66 & 70.9 & 1.1779 \\
\hline 3 & 28.57 & 64.3 & 1.7298 \\
\hline 4 & 50.0 & 52.4 & 2.5209 \\
\hline 5 & 66.66 & 43.1 & 2.5239 \\
\hline 6 & 75.00 & 38.5 & 2.4766 \\
\hline 7 & 80.00 & 35.72 & 2.6242 \\
\hline
\end{tabular}

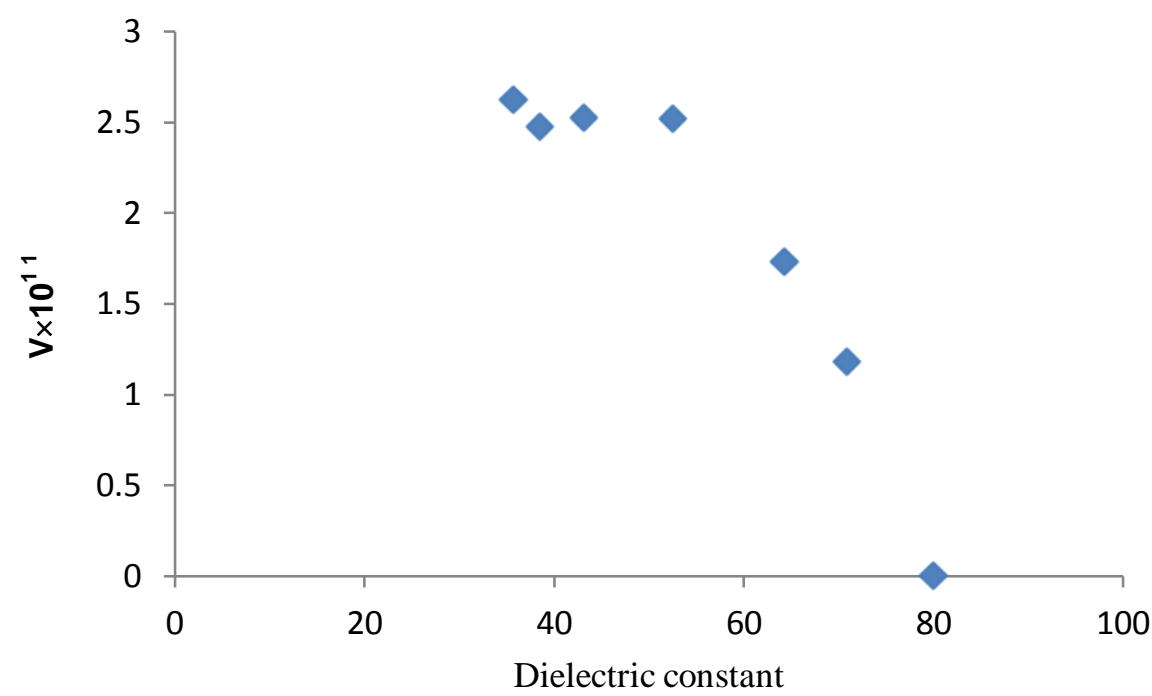

Figure 2a. Dielectric constant of the aqueous-ethanol solvent mixture versus velocity of $\mathrm{NaCl}$ precipitation.

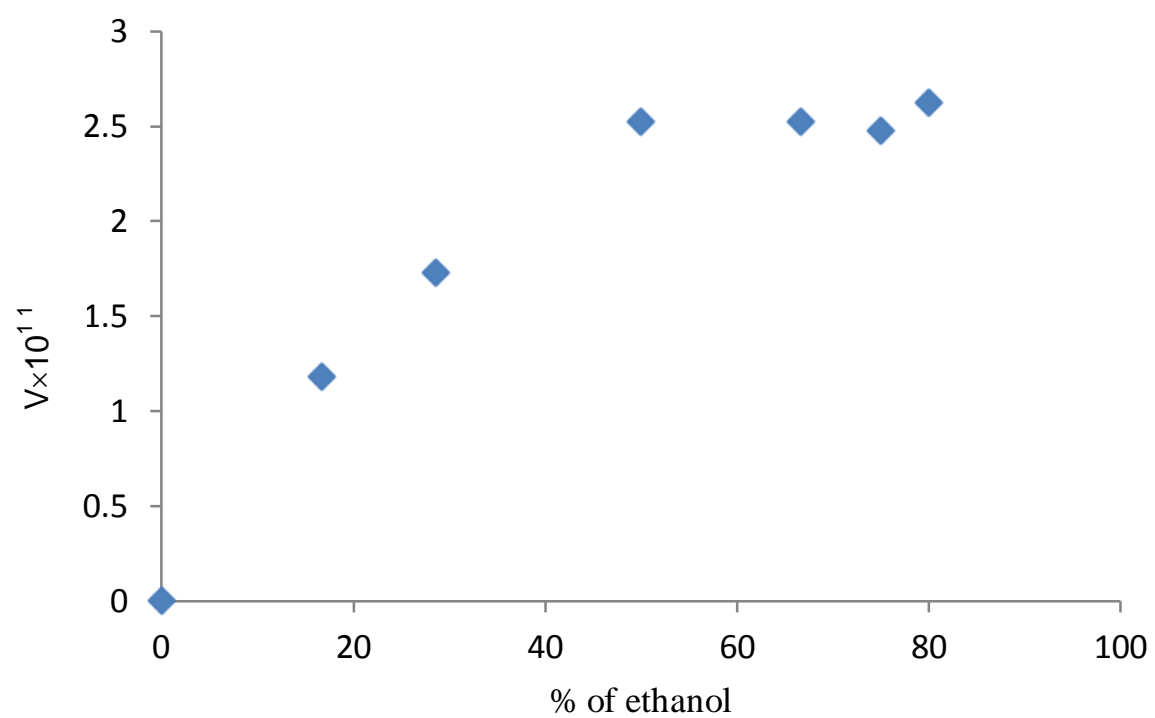

Figure 2b. Percent of ethanol in aqueous-ethanol solvent mixture versus velocity of $\mathrm{NaCl}$ precipitate. 
Table 4. Calculated magnitude of super saturation by Equation (4).

\begin{tabular}{cccc}
\hline No. & \% Ethanol & Dielectric constant & $\frac{\boldsymbol{M}_{\boldsymbol{i}}-\boldsymbol{M}_{f}}{\boldsymbol{M}_{f}}$ \\
\hline 1 & 0 & 80.0 & 0.00 \\
2 & 16.66 & 70.9 & 0.11 \\
3 & 28.57 & 64.3 & 0.21 \\
4 & 50.0 & 52.4 & 0.57 \\
5 & 66.66 & 43.1 & 1.19 \\
6 & 75.00 & 38.5 & 2.13 \\
7 & 80.00 & 35.72 & 16.46 \\
\hline
\end{tabular}

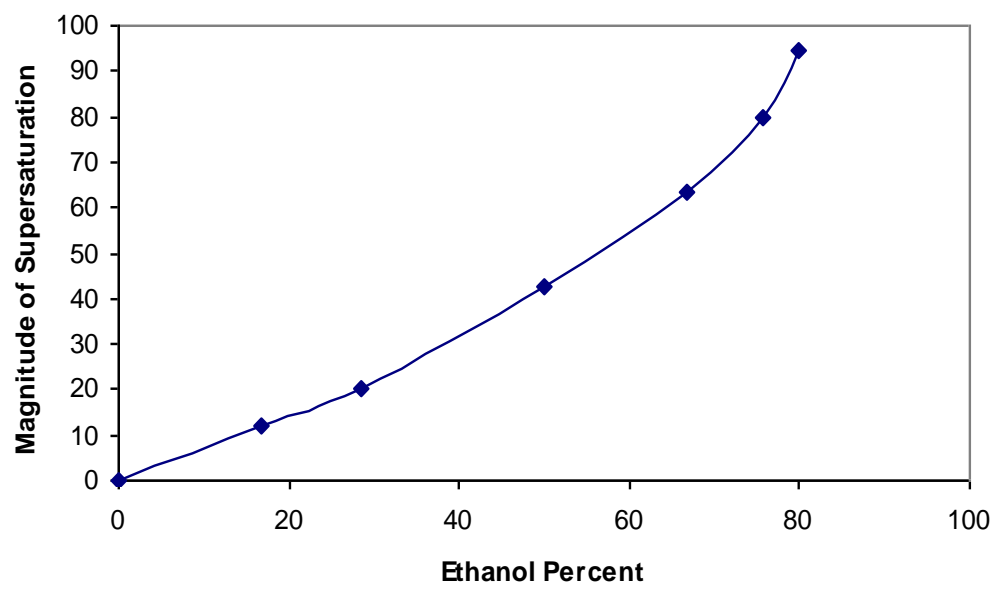

Figure 3a. Percent of ethanol versus magnitude of super saturation.

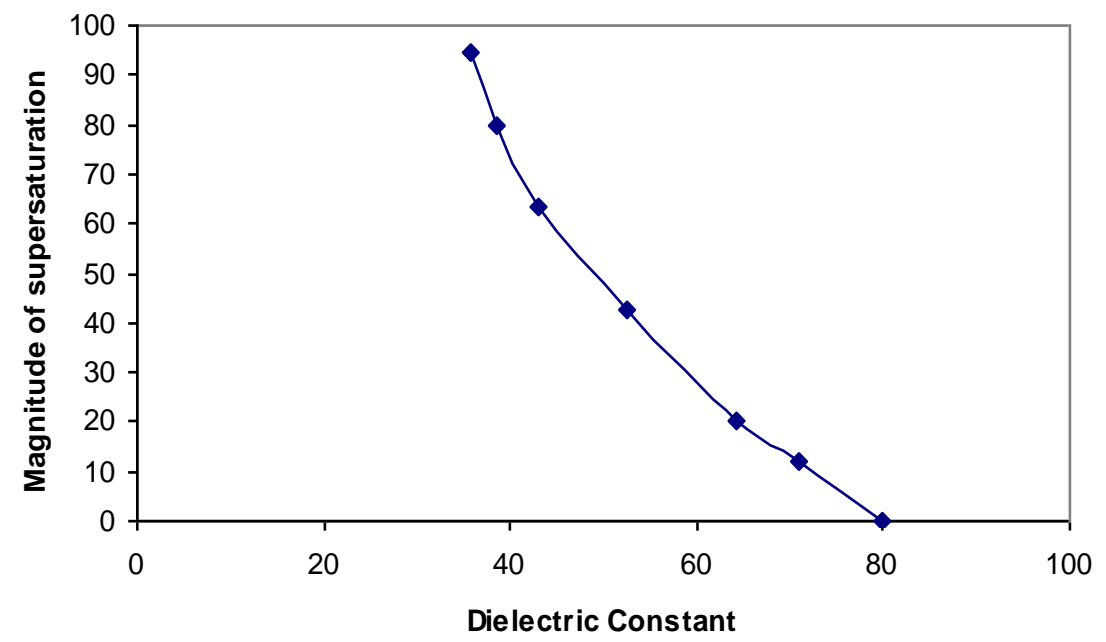

Figure 3b. Dielectric constant versus magnitude of super saturation.

of induced supersaturation, is calculated by Equation (5):

$$
\frac{M_{i}-M_{f}}{M_{f}}
$$

where, $M_{i}$ and $M_{f}$ are the initial and final (solubility) solution concentration, respectively for each experiment.

Table 4 and Figure $3 a$ and $b$ represent the results obtained. 


\section{Conclusion}

The induced super saturation of a semi saturated $\mathrm{NaCl}$ aqueous solutions was studied upon addition of $96 \%$ ethanol and correlated to the average dielectric constant of the aqueous-ethanol mixture. It was evident that the solubility of the salt is connected with the dielectric constant of the medium. The velocity of crystal growth was found to increase with the lowering of the dielectric constant up to a value of 52.0 and remains almost constant below that value.

A magnitude of supersaturation was introduced as a measure of precipitation.

\section{ACKNOWLEDGEMENT}

This project was supported by King Saud University, Deanship of Scientific Research, College of Science Research Center.

\section{REFERENCES}

Al-Jibbouri S, Ulrich J (2001). The Influence of Impurities on the Crystallization Kinetics o sodium chloride. Cryst. Res. Technol., 3: 1365.

Al-Jibbouri S, Ulrich J (2002). Kinetics and Mechanism of the Crystallization Process. Cryst. Growth, pp. 234-237.

Birchall JD, Davey RJ (1981). The crystallization of sodium chloride from aqueous Solution in the presence of polysaccharides. J. Cryst. Growth, 54(2): 323-329.

Doki N, Kubota N, Yokota M , Kimura S, Sasaki S (2002). Production of Sodium chloride crystals of uni-modal size distribution by Batch Dilution crystallization. J. Chem. Eng. Jpn., 35: 1099.

Kubota N, Otosaka H, Doki N, Yokota M, Sato A (2000). Effect of lead(II) impurity on the growth of sodium chloride. J. Cryst. Growth, 220: 135.

Linnikov OD (2006a). Spontaneous crystallization of potassium chloride from aqueous and aqueous -ethanol solutions. Cryst. Res. Technol., 41(1): 10-17.

Linnikov OD (2006b). Spontaneous crystallization of sodium chloride from aqueous and aqueous -ethanol solutions Part 2. ibid, (2): 138144.
Linnikov OD (2007). Spontaneous Crystallization of Sodium chloride from aqueous and aqueous -ethanol solutions Part 3. ibid, 42(8): 758-765.

Linnikov OD (2004). Spontaneous crystallization of potassium chloride from aqueous and aqueous-ethanol solutions Part 2. Mech. of aggregation coalescence crystals. Ibid, 39(6): 529-539.

Mohameed UH, Zhang SB, Yuan JJ (1997). Influence of the pH -value on the growth dissolution rate of potassium chloride. Bull. Soc. Sea Water Sci. Jpn., 51: 973.

Polak AF (1966). Hardening of Mono-mineral Astringent Substances. Publishers literature constructions, pp. 273- 278

Ramana VV, Malakondaiah K (2010). PC Based Instruments for the Measurement of Dielectric Constant of Liquids. Sensors Transducer J., 112: 73-79.

Smoluchowski MV (1917). Trying a Mathematical Theory for the Kinetics of Solution co-agulation. Z. Phys. Chem., 92: 129. 